- Case Report

\title{
Radiolucent Pure Matrix Stones on Computed Tomography Scan, Arising in Patient with Type I Diabetes and Chronic Kidney Disease: A Case Report
}

\author{
Young-Woong Song',*, Ju-heon Kim², Kitae Bang', Jong Ho Shin', Kyeong Min Kim', Jinuk Jeong' \\ 'Department of Internal Medicine, Eulji University School of Medicine, Daejeon, Korea \\ ${ }^{2}$ Department of Pathology, Eulji University School of Medicine, Daejeon, Korea
}

Kidney matrix stones are a rare form of calculi, which are challenging to diagnose. Matrix stones consist of a proteinaceous material which has a radiolucent appearance that might be overlooked on imaging. Recently, endourological intervention has been the standard treatment method for matrix stones. We report a case of urinary matrix stones in a patient with type 1 diabetes mellitus and chronic kidney disease, in whom the stones formed into a pure matrix and were not visualized in the computed tomography scan. The stones were found after additional work-up, and they were managed using a transureteral stone basket, not through endourological intervention.

Keywords: Urolithiasis; Matrix Stone; Radiolucent; Diabetic Nephropathies; Case Report

Received: April 23, 2020, Revised: May 25, 2020, Accepted: May 27, 2020

*Corresponding Author: Young-Woong Song https://orcid.org/0000-0002-7848-0919

Tel: +82-31-8041-3668, Fax: +82-31-8041-3668, E-mail: herocode1@gmail.com 


\section{INTRODUCTION}

Urinary matrix stones are a rare form of urinary calculi that are typically radiolucent. ${ }^{1)}$ However, the common ureteral stones are easily detected using computed tomography (CT) scanning. Thus, patients complaining of flank pain with radiolucent stones on CT may be misdiagnosed with myalgia. A skilled physician may certainly suspect tumors or uric acid stones, although there should be a high index of suspicion for a matrix stones in such cases. Matrix stones usually occur in individuals with diabetes mellitus (DM) or chronic kidney disease (CKD), and these radiolucent concretions are composed primarily of a non-crystalline muco-protein matrix. ${ }^{2)}$ We report a case of matrix stones in a patient with type I DM and CKD, in whom the stones caused unilateral ureteral obstruction. This resulted in an acute kidney injury which required renal replacement therapy.

\section{CASE REPORT}

In May 2016, a 24-year-old woman with type 1 DM and CKD was admitted to the emergency room with abrupt-onset right flank pain. Laboratory tests at admission revealed an elevated creatinine (Cr) level (last recorded Cr, 1.73 mg/dL; Cr on admission, 3.39 mg/dL). Mild hydronephrosis was diagnosed through CT, but ureter calculi were not visualized (Figure 1). A Technetium-99m (Tc-99m) diethylene-triamine-pentaacetate renal scan was performed to determine differential diagnoses for acute hydronephrosis (Figure 2). Decreased right renal flow was observed, and percutaneous nephrostomy was performed for the right kidney. Urine was drained via nephrostomy, and gradual $\mathrm{Cr}$ decline was subsequently observed. Follow-up tubography revealed proximal ureteral obstruction and multiple filling defects (Figure 3).

We performed a ureter biopsy for confirmation, revealing a non-calcium-based acellular proteinaceous ureter stone (Figure 4). Due to the
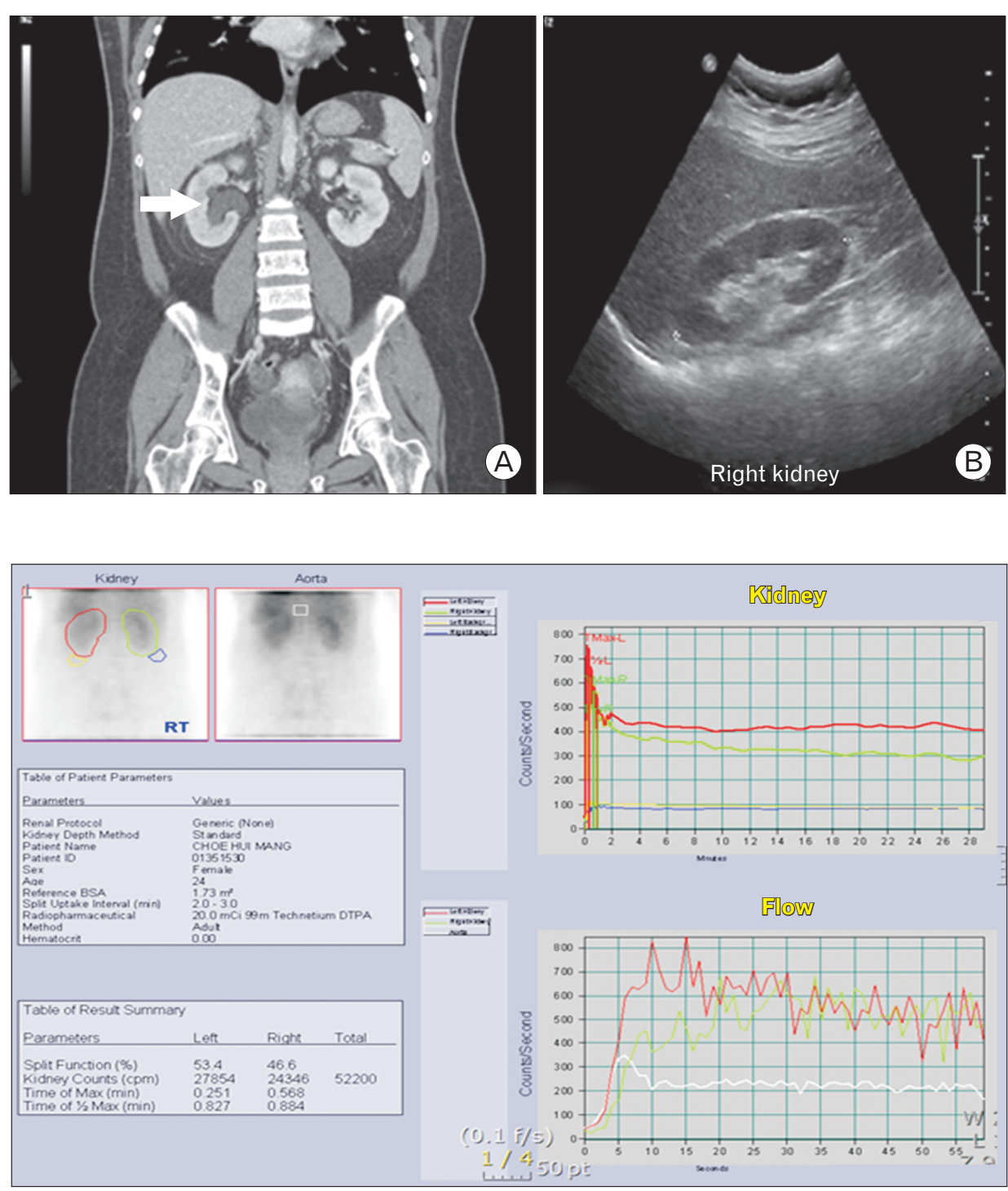

Figure 1. Abdomen computed tomography (A) shows new-onset hydronephrosis (arrow) in the right kidney compared with previous abdominal ultrasonography (B).
Figure 2. Decreased right kidney flow is observed on diethylene-triamine-pentaacetate renal scan. 
small stone size, transureteral stone extraction was performed using a basket device rather than via percutaneous nephrolithotomy (PCNL) (Figure 5). The fully extracted stone was a soft, fragile, reddish mass (Figure 6).

\section{DISCUSSION}

Matrix stones, also known as fibrinomas, colloid calculi, or albumin calculi, are a rare form of calculi first described by Gage and Beal ${ }^{3)}$ in 1908. There is paucity of literature on matrix stones, mostly consisting of anecdotal case reports. Most matrix stones contain calcium crystals,

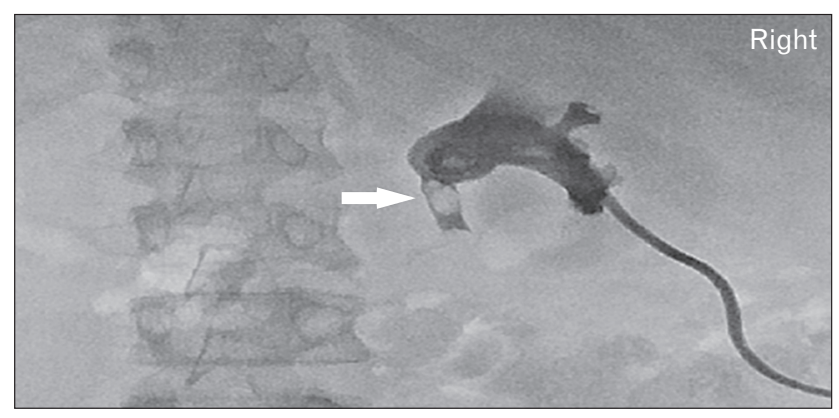

Figure 3. Tubography reveals filling defect (arrow) with proximal ureter obstruction.

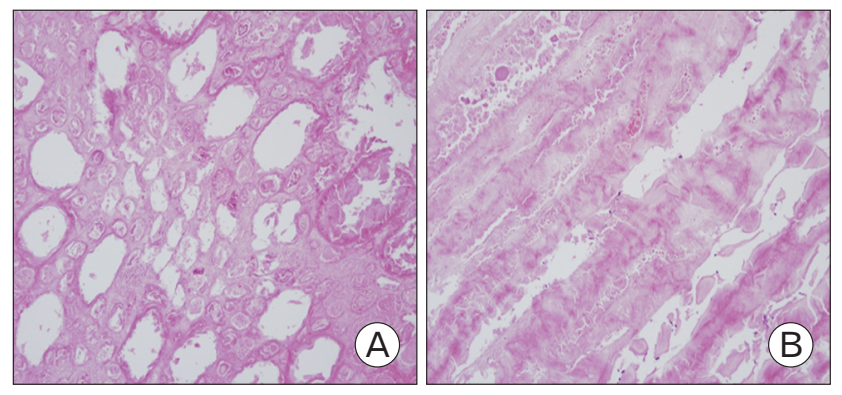

Figure 4. Ureter biopsy reveals a acellular homogenous and hyalinized stone in the ureter (A: H\&E stain) and an infarcted urothelial mucosa (B: H\&E stain). but the matrix component of a calcium-based stone comprises only $2.5 \%$ of its dry weight. Contrastingly, pure matrix stones may contain up to $65 \%$ protein, ${ }^{4)}$ and it was determined that the composition of matrix stones was approximately two-thirds mucoprotein and onethird mucopolysaccharide by weight. The main carbohydrate components were hexose and hexosamine, whereas threonine, leucine, serine, tyrosine, arginine, and lysine were the most common amino acids in the protein component. ${ }^{4)}$ Most matrix stones with even a small calcium-based component can be diagnosed using radiographic studies; however, $25 \%$ of matrix stones do not contain calcium-based material, resulting in a radiolucent appearance on imaging. Although the stones were not analyzed in this study, considering biopsy findings (i.e., an acellular and hyalinized stone without detectable calcium crystals) and a radiolucent filling defect on $\mathrm{CT}$, the stones in the present case may be considered to have a pure matrix component. It is unclear why some matrix calculi failed to fully calcify.

The radiographic diagnosis of urinary matrix calculi represents a diagnostic dilemma due to their variable appearance on imaging. Thus, matrix stones should be included in the differential diagnoses for filling defects of the urinary tract. Other differentials include blood clots, tumors, polyps, small uric acid or cystine stones, varices, ureteritis cystica, and sloughed renal papillae. ${ }^{1)}$

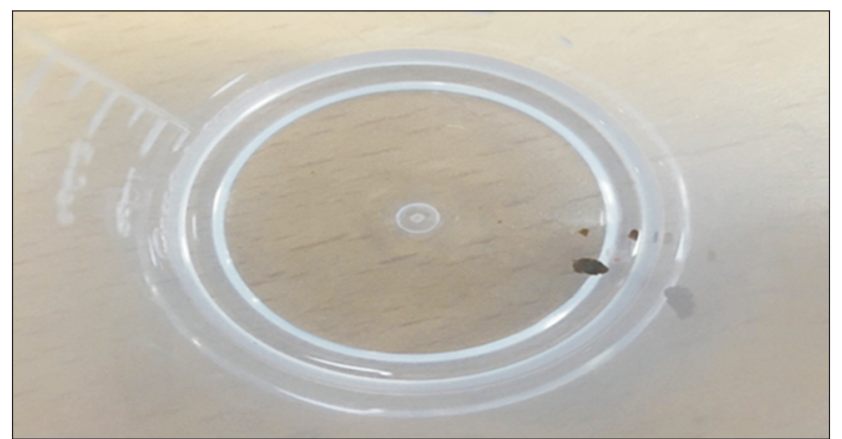

Figure 6. Macroscopic appearance of a stone following basket stone extraction.
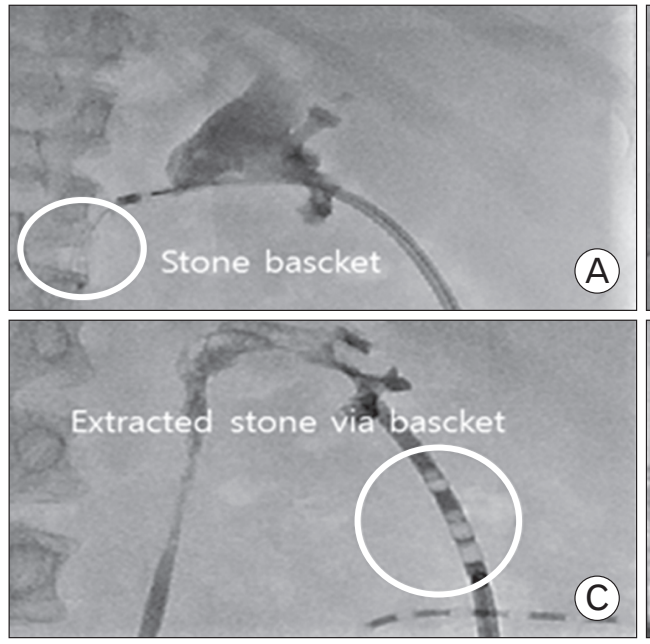

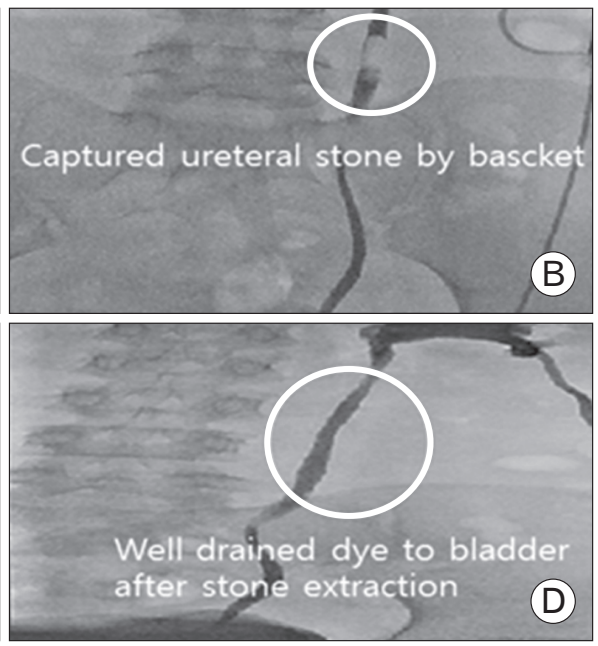

Figure 5. (A-D) Ureter stone extraction via stone basket. 
The etiology of matrix stones has not been elucidated. Calcigerous renal calculi are more common in men, whereas matrix stones are more common in women. Most studies have suggested that matrix stones were secondary to a urinary system infection, particularly of Proteus mirabilis or Escherichia-coli, ${ }^{5)}$ and mainly occur in diabetic patients for reasons as follows: (1) slow ureteral peristalsis and increased glomerular permeability in diabetic patients result in excessive protein and glucose in their urine, making it an ideal medium for bacterial growth; and (2) diabetic patients are immunocompromised and prone to urinary tract infections.

Similarly, patients with CKD, especially those undergoing dialysis, may have an increased risk of matrix stone formation. A possible mechanism is to speculate that the prolonged stagnation of urine with a high protein content, perhaps secondary to continued reabsorption of sodium and water, provides a nidus for stone formation. ${ }^{6)}$ Various proteins have been shown, including microfibrillar proteins and $\beta-2$ microglobulin. ${ }^{6)}$

Open surgery was formerly the preferred technique for urinary stone removal. ${ }^{7)}$ Some researchers have described the successful removal of matrix stones using a ureteroscope, in which PCNL affords the best probability of complete removal of matrix stones. ${ }^{8)}$ Additionally, although retrograde intrarenal surgery (RIRS) is often inadequate for large groups of stones, there have been notable reports of successful treatment with RIRS. ${ }^{9}$ However, shock wave lithotripsy is usually ineffective due to the gelatinous nature of matrix stones. ${ }^{2,8)}$ Antibiotic prophylaxis is believed to be effective in preventing matrix stone recurrences. ${ }^{10)}$ In our case, due to the small stone size, we attempted stone extraction using a stone basket rather than via PCNL. The transureteral stone basket device was inserted through nephrostomy to reach the stones, which were removed several times. It was less invasive than endourological treatment, and was highly successful.

Matrix stones can be challenging to diagnose as they mimic upper urinary tract diseases such as soft tissue masses. Therefore, they require a high index of suspicion. Matrix stones should be considered when a radiolucent filling defect in the upper urinary tract is present, particularly in DM or CKD patients with proteinuria. Although open surgery has been replaced by endourological intervention, the latter is still an invasive procedure. As matrix stones are typically soft and fragile, basket stone extraction can be an alternative treatment option for small matrix stones. Further investigations are necessary to generalize study findings and confirm this procedure as an alternative treatment method for matrix stones.

Occasionally, hydronephrosis is not clearly diagnosed. Hydronephrosis is less prominent in cases of incomplete obstruction of the ureter than in cases of complete obstruction, in which hydronephrosis can be easily observed. In our case, there was a debate among several physicians in various departments. We were initially hesitant to perform percutaneous nephrostomy due to its invasiveness and an un- clear diagnosis of hydronephrosis. We thus detected the matrix stones using tubography and successfully removed them. In our case, a mild hydronephrosis was suspected through comparisons with previous radiographs. Ambiguous hydronephrosis accompanied by abnormalities, such as abrupt onset flank pain, hematuria, changes in laboratory test results, and others, should be examined actively in patients at risk, even in the absence of previous radiographs. In addition to matrix stones, diseases such as tumors and hematomas may be associated with hydronephrosis, thus requiring thorough examinations.

\section{CONFLICT OF INTEREST}

No potential conflict of interest relevant to this article was reported.

\section{ORCID}

Young-Woong Song: https://orcid.org/0000-0002-7848-0919

Ju-heon Kim: https://orcid.org/0000-0002-3494-3301

Kitae Bang: https://orcid.org/0000-0002-8469-2010

Jong Ho Shin: https://orcid.org/0000-0002-8909-3404

Kyeong Min Kim: https://orcid.org/0000-0002-5414-4339

Jinuk Jeong: https://orcid.org/0000-0003-1786-0448

\section{REFERENCES}

1. Bani-Hani AH, Segura JW, Leroy AJ. Urinary matrix calculi: our experience at a single institution. J Urol 2005;173:120-3.

2. Shah HN, Kharodawala S, Sodha HS, Khandkar AA, Hegde SS, Bansal MB. The management of renal matrix calculi: a single-centre experience over 5 years. BJU Int 2009; 103:810-4.

3. Gage H, Beal HW. V. Fibrinous calculi in the kidney. Ann Surg 1908;48: 378-87.

4. Boyce WH, Garvey FK. The amount and nature of the organic matrix in urinary calculi: a review. J Urol 1956;76:213-27.

5. Wickham JE. Matrix and the infective renal calculus. Br J Urol 1975; 47:727-32.

6. Bommer J, Ritz E, Tschope W, Waldherr R, Gebhardt M. Urinary matrix calculi consisting of microfibrillar protein in patients on maintenance hemodialysis. Kidney Int 1979;16:722-8.

7. Stoller ML, Gupta M, Bolton D, Irby PB 3rd. Clinical correlates of the gross, radiographic, and histologic features of urinary matrix calculi. J Endourol 1994;8:335-40.

8. Rowley MW, Faerber GJ, Wolf JS Jr. The University of Michigan experience with percutaneous nephrostolithotomy for urinary matrix calculi. Urology 2008;72:61-4.

9. Chan CH, El-Hakim A, Andonian S. Renal matrix stone managed by ureteroscopic holmium laser lithotripsy. Can J Urol 2010;17:5127-30.

10. Miwa S, Yamamoto H, Sugata T. Antibiotics therapy was effective in preventing bilateral staghorn renal matrix stones. Urol Res 2011;39:6972. 